\title{
The prognostic significance of carcinoma- associated fibroblasts and tumor-associated macrophages in nasopharyngeal carcinoma
}

This article was published in the following Dove Press journal: Cancer Management and Research

\author{
Yahui Yu, ${ }^{1-3, *}$ Liangru Ke, ${ }^{1,4, *}$ \\ Xing Lv, ${ }^{1,2}$ Yi hong Ling, ${ }^{1,5}$ \\ Jiabin Lu, ${ }^{1,5} \mathrm{Hu}$ Liang, ${ }^{1,2}$ \\ Wenze Qiu, ${ }^{1,2}$ Xinjun \\ Huang, ${ }^{1,2}$ Guoying Liu, ${ }^{1,2}$ \\ Wangzhong $\mathrm{Li}^{1}{ }^{1,2}$ Xiang \\ Guo, ${ }^{1,2}$ Weixiong $X i a,{ }^{1,2}$ \\ Yanqun Xiang ${ }^{1,2}$
}

'Sun Yat-Sen University Cancer Center, State Key Laboratory of Oncology in

South China, Collaborative Innovation

Center for Cancer Medicine,

Guangdong Key Laboratory of

Nasopharyngeal Carcinoma Diagnosis

and Therapy, Guangzhou, Guangdong,

People's Republic of China;

${ }^{2}$ Department of Nasopharyngeal

Carcinoma, Sun Yat-Sen University

Cancer Center, Guangzhou,

Guangdong, People's Republic of

China; ${ }^{3}$ Department of Radiation

Oncology, Oncology Center, Zhujiang Hospital, Southern Medical University,

Guangzhou, Guangdong, People's Republic of China; ${ }^{4}$ Department of Diagnostic Radiology, Sun Yat-Sen University Cancer Center, Guangzhou, Guangdong, People's Republic of China; ${ }^{5}$ Department of Pathology, Sun Yat-Sen University Cancer Center, Guangzhou, Guangdong, People's Republic of China

*These authors contributed equally to this work

Correspondence: Weixiong Xia; Yanqun Xiang

Department of Nasopharyngeal

Carcinoma, Sun Yat-Sen University

Cancer Center, 65I Dongfeng Road East,

Guangzhou, Guangdong 510060, People's

Republic of China

Tel +862087343359

Email xiawx@sysucc.org.cn; xiangyq@

sysucc.org.cn
Purpose: Tumor stroma cells play an important role in the carcinogenesis and progression of cancer. The aim of the present investigation was to explore the predictive role of carcinomaassociated fibroblasts (CAFs) and tumor-associated macrophages (TAMs) in nasopharyngeal carcinoma (NPC).

Patients and methods: The densities of CAFs and TAMs were measured by immunohistochemistry staining for $\alpha$-smooth muscle actin ( $\alpha$-SMA), CD68, and CD163 in two sets of tissue microarrays including 260 pretreatment NPC tissues, that is, a training test comprising of 152 patients and a validation set comprising of 108 patients. Chi-square tests were performed for comparisons among the groups. Survival rates were estimated by using the Kaplan-Meier method and compared with log-rank tests. Cox proportional hazards models were used to identify significant independent variables.

Results: Patients older than 50 years showed a lower expression of CD68, and there was a positive relationship between the densities of CAFs and CD163+ TAMs $(p=0.001)$. In the multivariate analysis of the training test, both $\alpha$-SMA and CD163 were independent prognostic factors for overall survival and progression-free survival (all $p<0.05$ ). Based on the expression levels of $\alpha$-SMA and CD163, patients were categorized into three groups: high-risk, intermediate-risk, and low-risk groups according to both high, either high, and both low, respectively. Survival analysis and Cox multivariate analysis showed that the risk groups based on $\alpha$-SMA and CD163 expression were independent predictors for the survival of patients with NPC in the training test, which was also confirmed by the validation test.

Conclusion: A patient's risk group based on the level of CD163+ TAMs and CAFs was an independent predictor of survival, which may facilitate patient counseling and individualized treatment.

Keywords: CD68, CD163, $\alpha$-SMA, risk groups, multivariate analysis

\section{Introduction}

Nasopharyngeal carcinoma (NPC) is an endemic malignancy in Southern China and Southeast Asia that is highly invasive and has a high metastasis rate. ${ }^{1-4}$ With the development of diagnostic imaging, radiotherapeutic techniques, and chemotherapy regimens, the ability to control NPC has improved significantly. ${ }^{5}$ However, $20 \%-30 \%$ of newly diagnosed, non-metastatic patients still develop local recurrence or distant metastasis after radical chemoradiotherapy. ${ }^{6,7}$ Although the tumor-node-metastasis (TNM) cancer staging system summarized by the American Joint Committee on Cancer and the International Union for Cancer Control (AJCC/UICC) provides a useful benchmark for establishing a treatment strategy and estimating the prognosis, there remain large variations in the clinical outcomes of patients with the same stage 
who are undergoing similar treatment strategies. ${ }^{8}$ Therefore, the staging system is based only on the anatomical extent, which is not enough to evaluate the whole tumor status or guide therapy.

Recently, a growing amount of evidence has indicated that tumor stroma cells play an important role in the carcinogenesis and progression of cancer. ${ }^{9-11}$ Cancer-associated fibroblasts (CAFs) are primary components of tumor stroma cell populations, which are characterized by "de novo" expression of $\alpha$-smooth muscle actin ( $\alpha$-SMA) and are known to promote the growth and invasion of cancer cells by various mechanisms, such as growth factor and chemokine production, extracellular matrix remodeling, and so on, as well as aid in the suppression of the host immune response. ${ }^{12-15}$ Tumor-associated macrophages (TAMs) are also major stromal components within tumors that exist as a heterogeneous population of cells derived from monocytes and are polarized into two functionally distinct forms, M1 and M2, in response to tissue microenvironments and/or inflammatory status. ${ }^{16}$ CD68 has been widely used as a pan-macrophage marker in previous studies, ${ }^{17}$ and the correlations between $\mathrm{CD} 68^{+}$TAMs and the prognosis of patients were inconsistent among various cancers including thyroid cancer, lung cancer, hepatocellular cancer, esophageal cancer, oral squamous cell carcinoma (OSCC), and so on. ${ }^{18-22}$ CD163 is one of the markers used to identify M2 macrophages in several tumors and has predicted adverse outcomes. ${ }^{23-29}$ Some reports have indicated that CAFs and TAMs are synergistically associated with the prognostic significance in several types of tumors. ${ }^{30-32}$ However, the clinical significance of TAMs and CAFs and the relationship between TAMs and CAFs in NPC has not been determined.

In this study, initially, we used triplets of tissue microarrays (TMAs), including 152 NPC specimens from patients with long follow-ups to clarify the clinical significance of TAMs and CAFs by analyzing the expression of $\alpha$-SMA (fibroblasts), CD68, and CD163 and their relationship between one another. Then, we constructed a model based on these markers to predict NPC patients' prognosis, which was further validated with another TMA comprising 108 NPC specimens.

\section{Patients and methods Patients and samples}

We performed a retrospective review of clinical records of patients with newly diagnosed, non-metastatic NPC in Sun Yat-Sen University Cancer Center (SYSUCC), and those with high-quality, formalin-fixed, paraffin-embedded NPC tissues from the original diagnostic biopsy were enrolled in the study.
Two sets of TMAs including 260 specimens were analyzed in our study. TMA comprising specimens from 152 patients treated with two-dimensional radical radiotherapy (2DRT) between November 1999 and December 2000 was used as the training set, and another TMA comprising specimens from 108 patients treated with intensity-modulated radiation therapy (IMRT) between December 2010 and October 2013 was used for validation. All patients were restaged according to the 7 th edition UICC/AJCC cancer staging system. This study was reviewed and approved by the institutional review board and ethics committee of SYSUCC. Informed written consents for using tissue and clinical data for scientific research were obtained from all participants in our study. TMA was constructed as described previously. ${ }^{33}$

\section{Immunohistochemistry (IHC)}

Briefly, 4- $\mu \mathrm{m}$ thick tissue sections of formalin-fixed, paraffinembedded NPC tissues were deparaffinized in xylene and rehydrated through graded alcohol. Then, antigenic retrieval was performed with sodium citrate and a high-pressure boiler for 20 minutes. After cooling down to the room temperature, the sections were incubated with $3 \%$ hydrogen peroxide for 10 minutes to inhibit endogenous peroxidases. Then, the sections were incubated with anti-CD68 (diluted 1:80; Boster, Wuhan, China), anti-CD163 (diluted 1:100; Zhongshan Golden Bridge, Beijing, China), and anti- $\alpha$-SMA (diluted 1:200; Zhongshan Golden Bridge) antibodies overnight at $4^{\circ} \mathrm{C}$. The primary antibodies were detected by an EnVision kit (DAKO, Carpinteria, CA, USA) according to the manufacturer's instruction.

\section{IHC assessment}

Each section was screened at low-power magnification to identify the areas with the highest staining density. The positive expression of CD68 or CD163 was defined by a granular cytoplasm or a cytoplasmic and membrane staining pattern. In addition, the positive expression of $\alpha$-SMA was defined by the staining of large spindle-shaped fibroblasts around the tumor cells, and vascular-positive staining as an internal control was not classified. The expression of $\alpha-S M A, C D 68$, and CD163 was classified into four grades: negative (0), no staining; scant (1), a small amount of scattered staining; focal (2), concentrated stains with an irregular and non-continuous focus; and abundant (3), concentrated stains with an extensive and continuous focus; this classification system was similar to the system used in a previous study. ${ }^{31}$ Each section was scored independently by two pathologists. The average values from the two pathologists were used for further analysis. 


\section{Statistical analysis}

Chi-square tests were performed for comparisons between groups. Spearman's correlation was used to analyze the correlation between the TAM and CAF markers. Survival rates were estimated by using the Kaplan-Meier method and compared with log-rank tests. Hazard ratios (HRs) and 95\% CIs were estimated by a multivariable Cox proportional hazards model. The survival duration was calculated from the first day of NPC diagnosis. The primary endpoint was overall survival (OS), which was calculated from the date of diagnosis to the date of death. The secondary endpoints were progression-free survival (PFS), distant metastasis-free survival (DMFS), and locoregional failure-free survival (LRFFS).

All statistical tests were two sided, and $p$-values $<0.05$ were considered to be statistically significant. All statistical analyses were performed by using SPSS version 22.0 (IBM Corporation, Armonk, NY, USA).

\section{Results}

\section{Patient characteristics and treatment in the training set}

The training set included 121 males and 31 females with a median age of 48 years (range, 18-71 years). There were $4(2.6 \%)$ patients with stage I disease, $56(36.8 \%)$ patients with stage II disease, 54 (35.5\%) patients with stage III disease, and 38 (25\%) patients with stage IVA-B disease. All patients underwent 2DRT with a daily fraction of 2.0 Gy and 5 fractions per week; the radiotherapy dose ranges to the nasopharynx and neck were 60-80 Gy and 50-70 Gy, respectively. Among the 152 patients with a median followup time of 130 months (range, 5-144 months), 70 (46.1\%) patients died as a result of NPC, and 5 (3.3\%) patients died of a non-cancer-related disease. Thirty-two (21.1\%) patients suffered from locoregional recurrence, and 33 (21.7\%) patients suffered from distant metastasis. The 10-year OS, PFS, LRFFS, and DMFS rates were $48 \%, 55 \%, 76 \%$, and $76 \%$, respectively.

\section{CD68, CDI63, and $\alpha-S M A$ in NPC specimens}

According to the expression levels of CD68, CD163, and $\alpha$-SMA, patients were allocated into the "high" group for scores of 2-3 and the "low" group for scores of 0-1 (Figure 1). The distributions of the characteristics of the training sets within the scoring groups are summarized in Table 1. There were no significant relationships among the expression levels of CD68, CD163, and $\alpha$-SMA and the

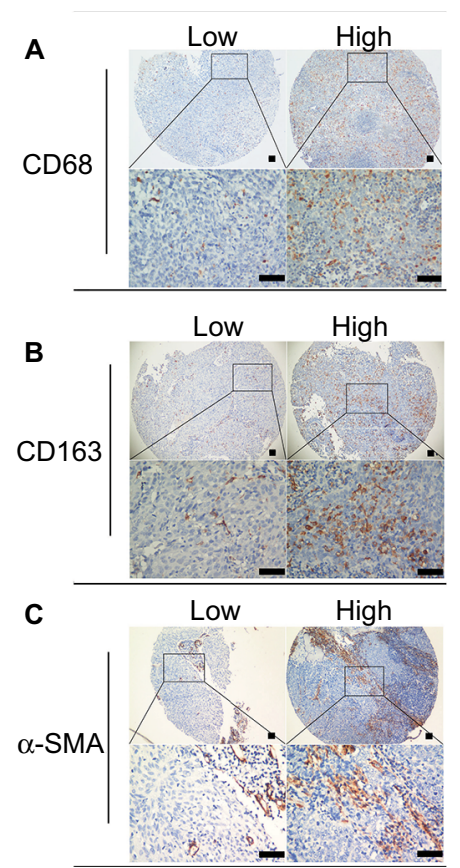

Figure I Representative samples of low and high expression of CD68 (A), CDI63 (B), and $\alpha$-SMA (C) in nasopharyngeal carcinoma (NPC) tissues detected by immunohistochemical staining. Blood vessels were used as internal positive controls for $\alpha$-SMA. Positively stained cells were identified as brown. Scale bar, $50 \mu \mathrm{m}$.

$\mathrm{T}$ stages, $\mathrm{N}$ stages, or clinical stages. Notably, there was a lower expression of CD68 in patients older than 50 years than in the younger patients, and patients in the group of low CD163 expression tended to not undergo chemotherapy. Spearman's correlation revealed a direct correlation between CD163+ TAMs and CAFs (Table 2).

\section{Survival analysis and prognostic factors in NPC patients}

We examined the relationships among the density of TAMs and CAFs and patient survival rates in the training set. The 10-year OS, PFS, and LRFFS rates in the CD68 $8^{\text {high }}$ group $(\mathrm{n}=81)$ were $58 \%, 64 \%$, and $83 \%$, which were significantly higher than those of $37 \%, 45 \%$, and $67 \%$ in the CD68 $8^{\text {low }}$ group ( $\mathrm{n}=71 ; p=0.023, p=0.009$, and $p=0.031$ ), respectively. However, no significant difference in DMFS was observed between the two groups ( $p=0.142$; Figure 2 ). In contrast, the 10-year OS, PFS, LRFFS, and DMFS rates in the CD163 $3^{\text {high }}$ group $(\mathrm{n}=76)$ were $40 \%, 43 \%, 65 \%$, and $69 \%$, which were significantly lower than those of $57 \%, 68 \%, 86 \%$, and $84 \%$ in the $\mathrm{CD} 163^{\text {low }}$ group ( $\mathrm{n}=76 ; p=0.046, p=0.001, p=0.015$, and $p=0.035$ ), respectively (Figure 3 ). Moreover, the 10 -year OS, PFS, and LRFFS showed no significant differences between the $\alpha$-SMA ${ }^{\text {high }}$ group and the $\alpha$-SMA ${ }^{\text {low }}$ group. Only the 10 -year DMFS rate in the $\alpha$-SMA ${ }^{\text {low }}$ group was 
Table I Clinical characteristics of the I52 nasopharyngeal carcinoma patients in the training set

\begin{tabular}{|c|c|c|c|c|c|c|c|c|c|c|}
\hline \multirow{2}{*}{$\begin{array}{l}\text { Clinical } \\
\text { parameters }\end{array}$} & \multirow{2}{*}{$\begin{array}{l}\text { No. of } \\
\text { patients }\end{array}$} & \multicolumn{3}{|l|}{ CD68 } & \multicolumn{3}{|l|}{ CDI63 } & \multicolumn{3}{|l|}{$\alpha$-SMA } \\
\hline & & $\begin{array}{l}\text { Low } \\
(n=7 I)\end{array}$ & $\begin{array}{l}\text { High } \\
(n=8 I)\end{array}$ & $p$-value & $\begin{array}{l}\text { Low } \\
(n=76)\end{array}$ & $\begin{array}{l}\text { High } \\
(n=76)\end{array}$ & $p$-value & $\begin{array}{l}\text { Low } \\
(n=78)\end{array}$ & $\begin{array}{l}\text { High } \\
(n=74)\end{array}$ & $p$-value \\
\hline Gender & & & & 0.834 & & & 0.546 & & & 0.399 \\
\hline Female & 31 & 15 & 16 & & 17 & 14 & & 18 & 13 & \\
\hline Male & 121 & 56 & 65 & & 59 & 62 & & 60 & 61 & \\
\hline Age (years) & & & & 0.002 & & & 0.745 & & & 0.342 \\
\hline$\leq 50$ & 82 & 29 & 53 & & 40 & 42 & & 45 & 37 & \\
\hline$>50$ & 70 & 42 & 28 & & 36 & 34 & & 33 & 37 & \\
\hline T stage & & & & 0.870 & & & 0.620 & & & 0.446 \\
\hline TI-T2 & 91 & 43 & 48 & & 44 & 47 & & 49 & 42 & \\
\hline T3-T4 & 61 & 28 & 33 & & 32 & 29 & & 29 & 32 & \\
\hline $\mathrm{N}$ stage & & & & 0.219 & & & 0.276 & & & 0.599 \\
\hline No-NI & 110 & 48 & 62 & & 58 & 52 & & 55 & 55 & \\
\hline N2-N3 & 42 & 23 & 19 & & 18 & 24 & & 23 & 19 & \\
\hline Clinical stage & & & & 0.888 & & & 0.869 & & & 0.379 \\
\hline I-II & 63 & 29 & 34 & & 32 & 31 & & 35 & 28 & \\
\hline III-IV & 89 & 42 & 47 & & 44 & 45 & & 43 & 46 & \\
\hline Chemotherapy & & & & $0.78 \mid$ & & & 0.001 & & & 0.509 \\
\hline No & 105 & 50 & 55 & & 62 & 43 & & 52 & 53 & \\
\hline Yes & 47 & 21 & 26 & & 14 & 33 & & 26 & 21 & \\
\hline Vital status & & & & 0.023 & & & 0.035 & & & 0.145 \\
\hline Alive & 77 & 29 & 48 & & 45 & 32 & & 44 & 33 & \\
\hline Dead & 75 & 42 & 33 & & 31 & 44 & & 34 & 41 & \\
\hline
\end{tabular}

Table 2 Correlations among the expression levels of TAM and CAF markers in NPC patients

\begin{tabular}{llll}
\hline IHC markers & CD68 & CDI63 & a-SMA \\
\hline CD68 & $r=-0.092$ & $r=-0.038$ \\
& & $p=0.258$ & $p=0.644$ \\
CDI63 & & $r=-0.263$ \\
& & $p=0.001$ \\
\hline
\end{tabular}

Abbreviations: CAF, carcinoma associated fibroblasts; TAM, tumor-associated macrophages; NPC, nasopharyngeal carcinoma; IHC, immunohistochemistry; $\alpha$-SMA, $\alpha$-smooth muscle actin.

significantly higher than that in the $\alpha-\mathrm{SMA}^{\text {low }}$ group $(83 \%$ vs $69 \%, p=0.036$; Figure 4 ).

Then, a multivariate Cox proportional hazards model was used to discriminate the independent prognostic indicators for the OS and PFS. The expressions of CD163 and $\alpha-S M A$ were independent prognostic factors for the OS and PFS (all $p<0.05)$, the tumor $\mathrm{N}$ stage was significantly associated with PFS ( $p=0.013$ ), and age was an independent prognostic factor for OS $(p=0.049$; Table 3$)$.

\section{Failure risk groups based on CAF and TAM levels}

Considering the prognostic significance of the expression of both CD163 and $\alpha$-SMA, we categorized the patients into three failure risk groups based on their expression levels and compared the survival rates among the expression groups.
1) The high-risk group (high expression of both CD163 and $\alpha$-SMA) included $27(17.8 \%)$ patients; 2$)$ the intermediaterisk group (high expression of either CD163 or $\alpha$-SMA) included 96 (63.2\%) patients; and 3) the low-risk group (high expression of neither CD163 nor $\alpha$-SMA) included 29 (19.1\%) patients. The distributions of clinical characteristics in training set according to risk groups are summarized in Table 4. The 10-year OS rates for the high-, intermediate-, and low-risk groups were $33 \%, 52 \%$, and $69 \%$, respectively $(p=0.007)$. The 10-year PFS rates for the three groups were $29 \%, 53 \%$, and $85 \%$, respectively $(p<0.001)$. The 10 -year LRFFS rates for the three groups were $65 \%, 72 \%$, and $96 \%$, respectively ( $p=0.031$ ). In addition, the 10 -year DMFS rates were $48 \%, 80 \%$, and $89 \%$, respectively ( $p<0.001$; Figure 5 ). The multivariate analysis showed that the $\mathrm{N}$ stage and risk groups were independent prognostic factors of OS and PFS, and age was an independent prognostic factor of OS, which was also similar to previous multivariate analysis before grouping (Table 5).

\section{Validation of the CAF- and TAM-based grouping}

To confirm the prognostic significance of the failure risk grouping in patients treated with IMRT, the validation set of 108 NPC specimens were collected. All patients completed 
A
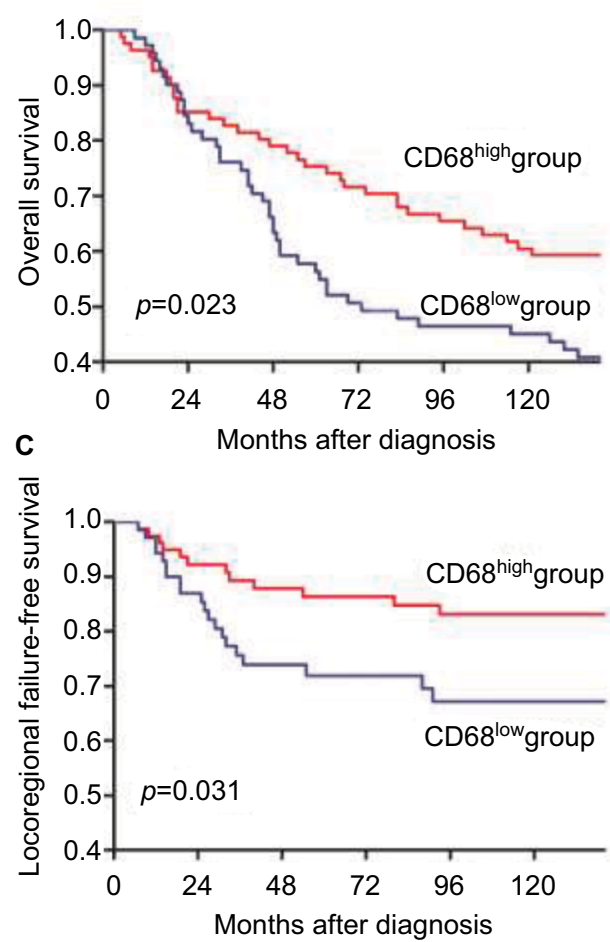

B
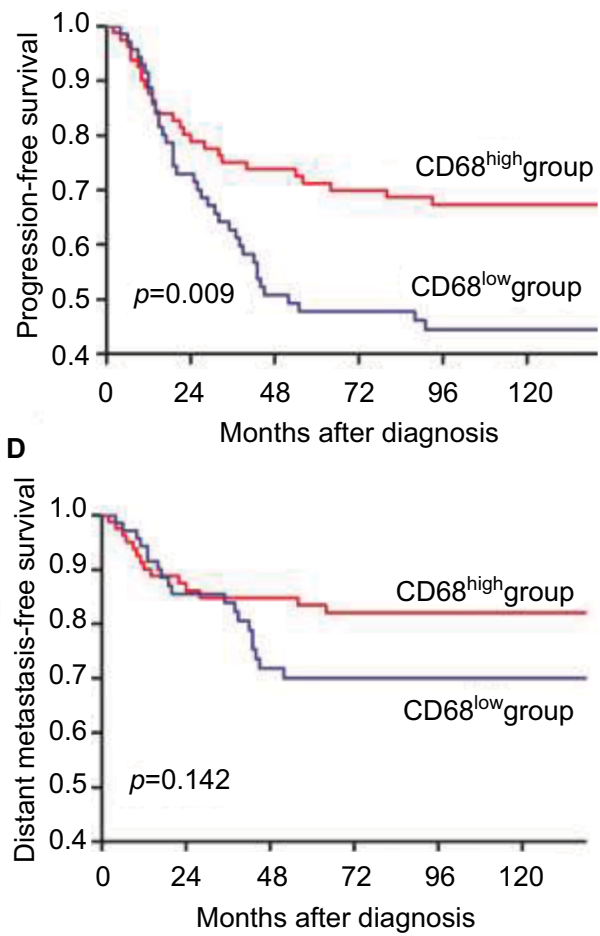

Figure 2 Kaplan-Meier analysis of the 10-year overall survival (A), 10-year progression-free survival (B), 10-year locoregional failure-free survival (C), and the 10-year distant metastasis-free survival (D) in relation to the expression of CD68 in the training test.

A
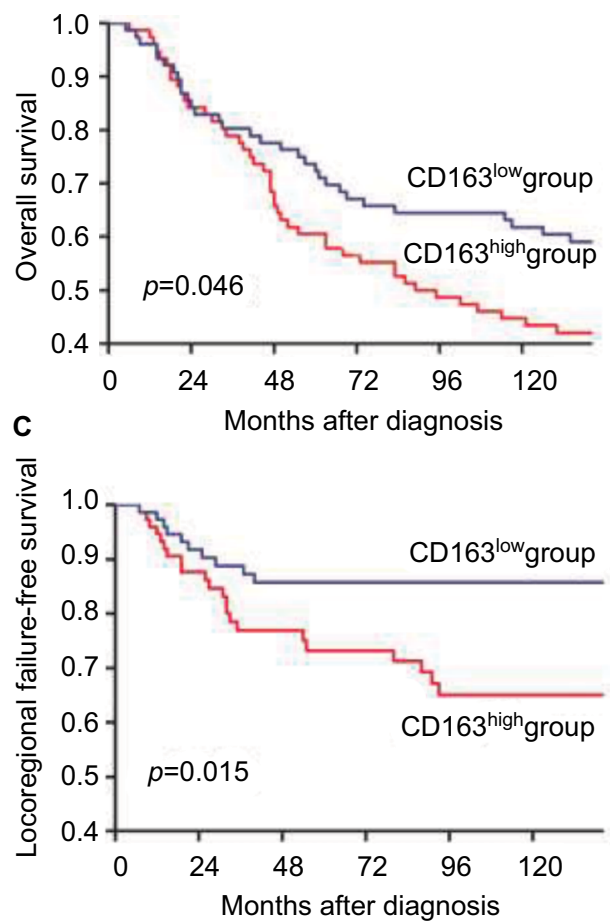

B

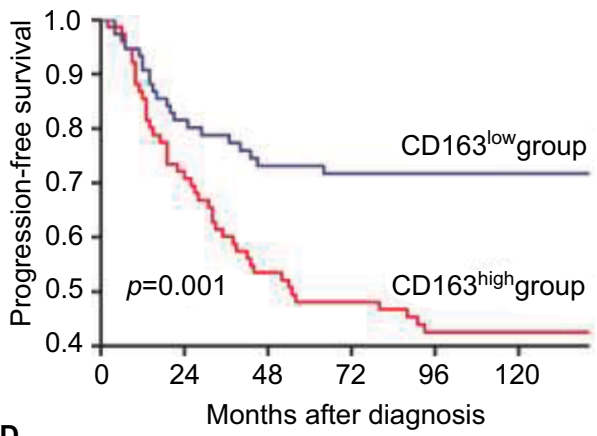

D

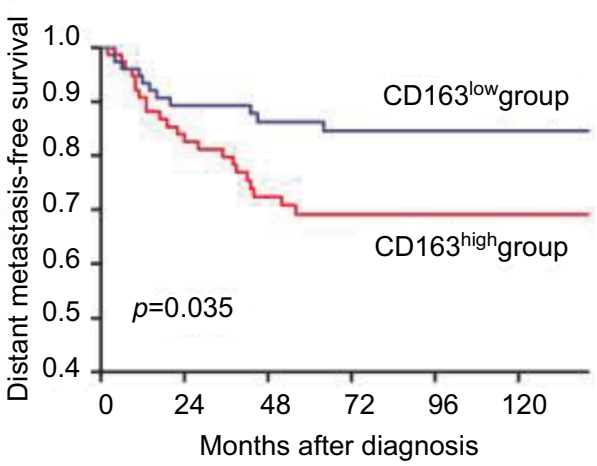

Figure 3 Kaplan-Meier analysis of the 10-year overall survival (A), 10-year progression-free survival (B), 10-year locoregional failure-free survival (C), and the 10-year distant metastasis-free survival (D) in relation to the expression of CDI63 in nasopharyngeal carcinoma tissues.

radical IMRT with a daily fraction of 2.0-2.33 Gy and 5 fractions per week; the radiotherapy dose ranges to the nasopharynx and neck were 70 and 60-66 Gy, respectively.
Among the 108 patients with a median follow-up time of 59 months (range, 4-88 months), 11 (10.2\%) patients died as a result of NPC, 8 (7.4\%) patients suffered from locoregional 
A

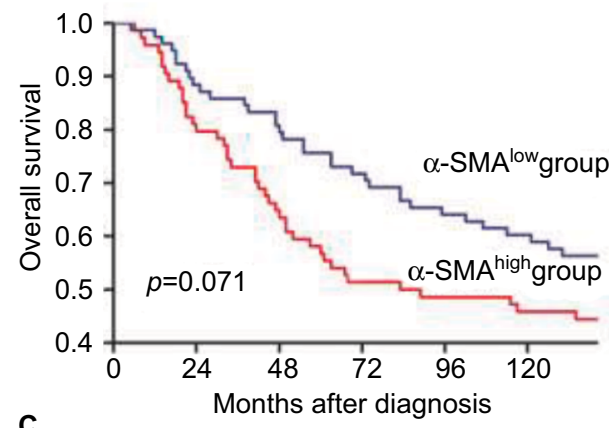

C

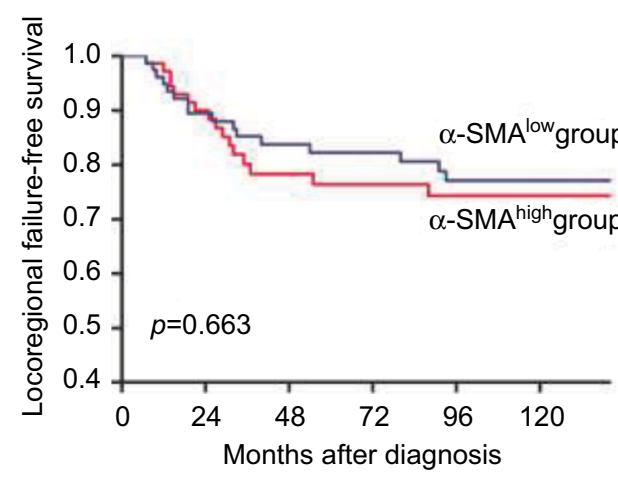

\section{B}

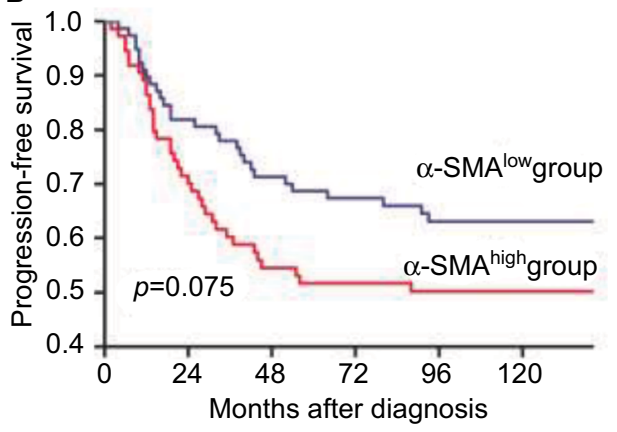

D

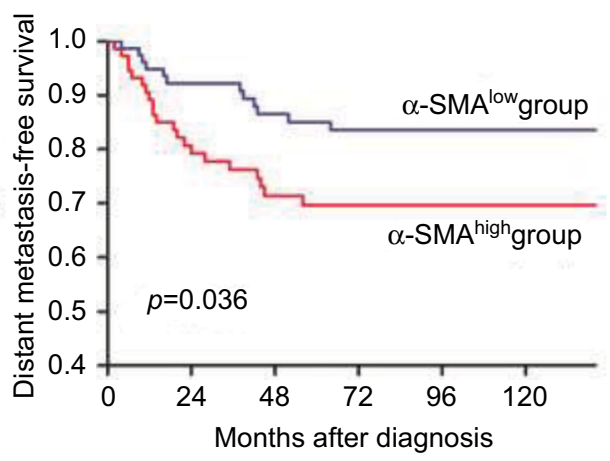

Figure 4 Kaplan-Meier analysis of the 10-year overall survival (A), 10-year progression-free survival (B), I0-year locoregional failure-free survival (C), and the I0-year distant metastasis-free survival (D) in relation to the expression of $\alpha$-SMA in the training test.

Table 3 Multivariate Cox proportional hazards model of the training set

\begin{tabular}{|c|c|c|c|c|}
\hline \multirow[t]{2}{*}{ Covariate } & \multicolumn{2}{|l|}{ Overall survival } & \multicolumn{2}{|c|}{ Progression-free survival } \\
\hline & HR (95\% Cl) & p-value & HR (95\% Cl) & $p$-value \\
\hline \multicolumn{5}{|l|}{ Gender } \\
\hline Female & Reference & & Reference & \\
\hline Male & $1.234(0.668-2.282)$ & 0.502 & $1.802(0.876-3.709)$ & 0.110 \\
\hline \multicolumn{5}{|l|}{ Age (years) } \\
\hline$\leq 50$ & Reference & & Reference & \\
\hline$>50$ & $1.628(1.002-2.644)$ & 0.049 & $1.294(0.760-2.203)$ & 0.343 \\
\hline \multicolumn{5}{|l|}{ T stage } \\
\hline T1-T2 & Reference & & Reference & \\
\hline T3-T4 & $1.760(0.787-3.935)$ & 0.169 & $1.572(0.674-3.666)$ & 0.295 \\
\hline \multicolumn{5}{|l|}{$\mathrm{N}$ stage } \\
\hline No-NI & Reference & & Reference & \\
\hline N2-N3 & $1.932(0.916-4.074)$ & 0.084 & $2.809(1.247-6.328)$ & 0.013 \\
\hline \multicolumn{5}{|l|}{ Clinical stage } \\
\hline I-II & Reference & & Reference & \\
\hline III-IV & $0.738(0.301-1.810)$ & 0.506 & $0.709(0.272-\mid .85 I)$ & 0.483 \\
\hline \multicolumn{5}{|c|}{ Chemotherapy } \\
\hline No & Reference & & Reference & \\
\hline Yes & $0.456(0.456-1.423)$ & 0.805 & $0.629(0.336-1.175)$ & 0.146 \\
\hline \multicolumn{5}{|l|}{ CD68 } \\
\hline Low & Reference & & Reference & \\
\hline High & $0.407(0.497-1.328)$ & 0.812 & $0.777(0.450-1.340)$ & 0.364 \\
\hline \multicolumn{5}{|l|}{ CDI63 } \\
\hline Low & Reference & & Reference & \\
\hline High & $1.848(1.114-3.064)$ & 0.017 & $2.956(1.664-5.252)$ & 0.001 \\
\hline \multicolumn{5}{|l|}{$\alpha$-SMA } \\
\hline Low & Reference & & Reference & \\
\hline High & $1.823(1.126-2.951)$ & 0.015 & $2.074(1.238-3.472)$ & 0.006 \\
\hline
\end{tabular}

Abbreviation: HR, hazard ratio. 
Table 4 Distribution of clinical characteristics of the whole cohort according to risk groups

\begin{tabular}{|c|c|c|c|c|c|c|c|c|c|c|}
\hline \multirow{2}{*}{$\begin{array}{l}\text { Clinical } \\
\text { parameters }\end{array}$} & \multicolumn{5}{|c|}{ Training set (risk groups) } & \multicolumn{5}{|c|}{ Validation set (risk groups) } \\
\hline & Case & Low & Intermediate & High & p-value & Case & Low & Intermediate & High & $p$-value \\
\hline Gender & & & & & $0.48 I$ & & & & & 0.272 \\
\hline Female & 31 & 8 & 19 & 4 & & 26 & 10 & 7 & 9 & \\
\hline Male & 121 & 21 & 77 & 23 & & 82 & 27 & 36 & 19 & \\
\hline Age (years) & & & & & 0.570 & & & & & 0.268 \\
\hline$\leq 50$ & 82 & 18 & 49 & 15 & & 70 & 27 & 28 & 15 & \\
\hline$>50$ & 70 & 11 & 47 & 12 & & 38 & 10 & 15 & 13 & \\
\hline T stage & & & & & 0.848 & & & & & 0.670 \\
\hline TI-T2 & 91 & 17 & 59 & 15 & & 12 & 4 & 6 & 2 & \\
\hline T3-T4 & 61 & 12 & 37 & 12 & & 96 & 33 & 37 & 26 & \\
\hline$N$ stage & & & & & 0.886 & & & & & 0.102 \\
\hline No-NI & 110 & 22 & 69 & 19 & & 49 & 17 & 15 & 17 & \\
\hline N2-N3 & 42 & 7 & 27 & 8 & & 59 & 20 & 28 & 11 & \\
\hline Clinical stage & & & & & 0.628 & & & & & 0.692 \\
\hline I-II & 63 & 13 & 41 & 9 & & 6 & 3 & 2 & 1 & \\
\hline III-IV & 89 & 16 & 55 & 18 & & 102 & 34 & 41 & 27 & \\
\hline Chemotherapy & & & & & 0.065 & & & & & 0.472 \\
\hline No & 105 & 25 & 64 & 16 & & 38 & 12 & 18 & 8 & \\
\hline Yes & 47 & 4 & 32 & 11 & & 70 & 25 & 25 & 20 & \\
\hline Vital status & & & & & 0.012 & & & & & 0.046 \\
\hline Alive & 77 & 21 & 47 & 9 & & 97 & 36 & 39 & 22 & \\
\hline Dead & 75 & 8 & 49 & 18 & & 11 & I & 4 & 6 & \\
\hline
\end{tabular}

A

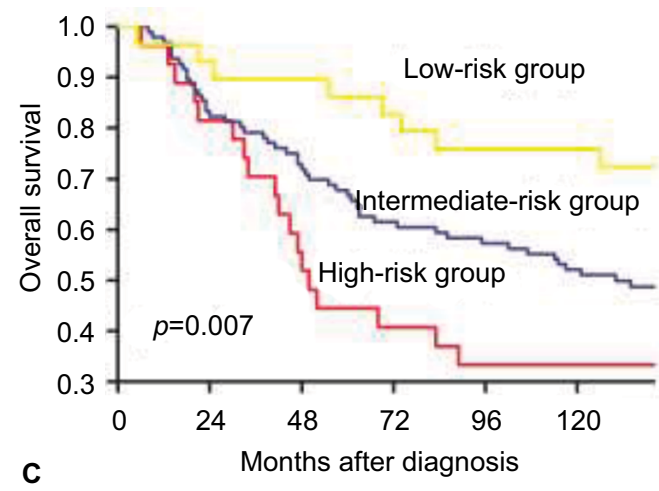

C

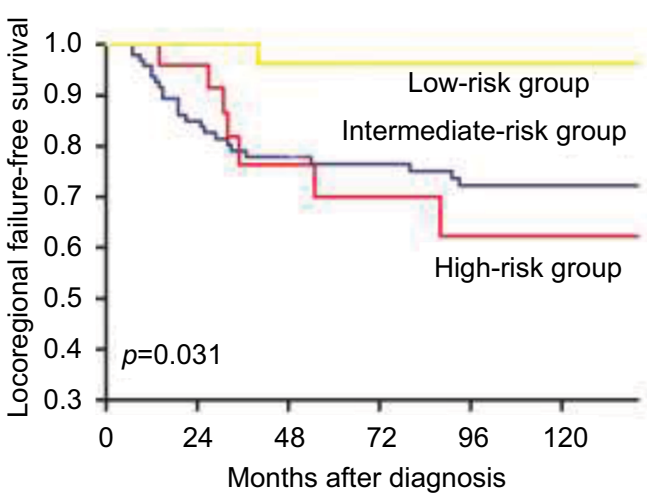

B

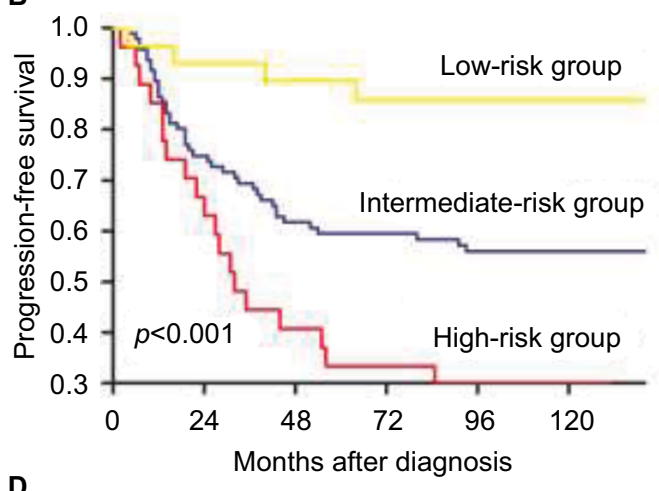

D

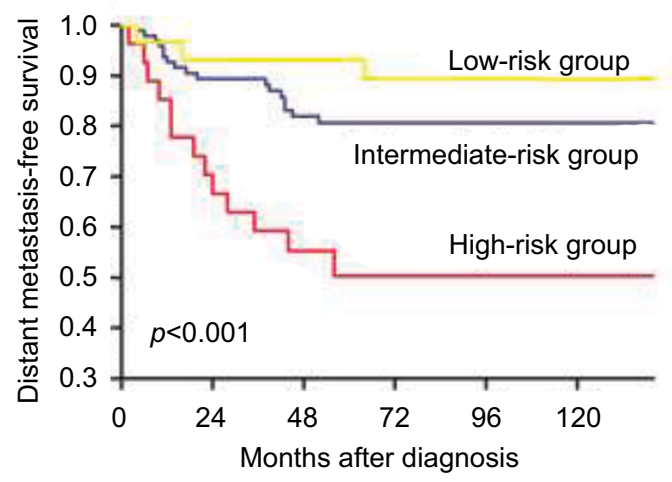

Figure 5 Kaplan-Meier analysis of the 10-year overall survival (A), 10-year progression-free survival (B), 10-year locoregional failure-free survival (C), and the I0-year distant metastasis-free survival (D) in relation to the risk groups of low-risk, intermediate-risk, and high-risk patients with nasopharyngeal carcinoma in the training test.

recurrence, and $11(10.2 \%)$ patients suffered from distant metastasis. The high-risk group, the intermediate-risk group, and the low-risk group included 37, 42, and 29 patients, respectively. The distributions of clinical characteristics in validation set according to risk groups are summarized in Table 4. The 5-year OS rates for the three groups were $76 \%$, 
Table 5 Multivariate Cox proportional hazards model of the cohort according to the risk groups

\begin{tabular}{|c|c|c|c|c|c|c|c|c|}
\hline \multirow[t]{3}{*}{ Covariate } & \multicolumn{4}{|l|}{ Training set } & \multicolumn{4}{|l|}{ Validation set } \\
\hline & \multicolumn{2}{|l|}{ Overall survival } & \multicolumn{2}{|c|}{ Progression-free survival } & \multicolumn{2}{|l|}{ Overall survival } & \multicolumn{2}{|c|}{ Progression-free survival } \\
\hline & HR (95\% Cl) & $p$-value & HR $(95 \% \mathrm{CI})$ & $p$-value & HR (95\% CI) & $p$-value & HR (95\% CI) & $p$-value \\
\hline \multicolumn{9}{|l|}{ Gender } \\
\hline Female & Reference & & Reference & & Reference & & Reference & \\
\hline Male & $1.22(0.66-2.26)$ & 0.517 & $1.78(0.89-3.65)$ & 0.117 & $1.41(0.35-5.68)$ & 0.627 & $3.42(0.74-15.83)$ & 0.117 \\
\hline \multicolumn{9}{|l|}{ Age (years) } \\
\hline$\leq 50$ & Reference & & Reference & & Reference & & Reference & \\
\hline$>50$ & $1.68(1.04-2.70)$ & 0.033 & $1.33(0.79-2.24)$ & 0.290 & $5.99(1.50-23.87)$ & 0.011 & $1.93(0.67-5.62)$ & 0.23 \\
\hline \multicolumn{9}{|l|}{ T stage } \\
\hline TI-T2 & Reference & & Reference & & - & - & - & - \\
\hline T3-T4 & $1.90(0.86-4.19)$ & 0.113 & $1.72(0.75-3.95)$ & 0.202 & - & - & - & - \\
\hline \multicolumn{9}{|l|}{$\mathrm{N}$ stage } \\
\hline No-NI & Reference & & Reference & & Reference & & Reference & \\
\hline N2-N3 & $2.08(1.01-4.28)$ & 0.047 & $3.07(\mathrm{I} .4 \mathrm{I}-6.68)$ & 0.005 & $3.17(0.82-12.29)$ & 0.096 & $2.05(0.68-6.21)$ & 0.206 \\
\hline \multicolumn{9}{|l|}{ Clinical stage } \\
\hline I-II & Reference & & Reference & & - & - & - & - \\
\hline III-IV & $0.70(0.29-1.70)$ & 0.430 & $0.67(0.26-I .7 I)$ & 0.404 & - & - & - & - \\
\hline \multicolumn{9}{|l|}{ Chemotherapy } \\
\hline No & Reference & & Reference & & Reference & & Reference & \\
\hline Yes & $0.78(0.45-1.36)$ & 0.385 & $0.64(0.34-1.19)$ & 0.150 & I.8I (0.44-7.42) & $0.4 I I$ & $2.93(0.78-10.99)$ & 0.111 \\
\hline \multicolumn{9}{|l|}{ Risk group } \\
\hline Low & Reference & & Reference & & Reference & & Reference & \\
\hline Intermediate & $2.25(1.05-4.82)$ & 0.037 & $4.08(1.44-11.55)$ & 0.008 & $2.90(0.3 \mathrm{I}-26.98)$ & 0.351 & $4.86(0.56-42.59)$ & 0.153 \\
\hline High & $3.85(1.64-9.04)$ & 0.002 & $8.70(2.89-26.15)$ & $<0.001$ & $9.63(1.07-87.06)$ & 0.044 & $21.23(2.62-171.94)$ & 0.004 \\
\hline
\end{tabular}

Abbreviation: HR, hazard ratio.

$89 \%$, and $97 \%$, respectively $(p=0.023)$. The 5 -year PFS rates for the three groups were $62 \%, 88 \%$, and $97 \%$, respectively $(p<0.001)$. The 5-year LRFFS rates for the three groups were $84 \%, 89 \%$, and $100 \%$, respectively ( $p=0.044)$. In addition, the 5 -year DMFS rates were $70 \%, 95 \%$, and $97 \%$, respectively $(p<0.001$; Figure 6). The multivariate analysis showed that risk groups were an independent prognostic factor of OS and PFS, and age was an independent prognostic factor of OS, which was similar to the results in training set (Table 5).

\section{Discussion}

To our knowledge, this is the first study to combine CAF and TAM markers to evaluate the prognosis of NPC patients. This study confirmed the prognostic value of TAMs and CAFs and the obvious correlation between the density of CAFs and M2 TAMs in NPC patients.

Previous studies have reported bidirectional correlations between patient survival and macrophage levels in many malignancies. For CD68, a high density of $\mathrm{CD}^{+} 8^{+}$macrophages was correlated with a decreased PFS and an increased likelihood of relapse in classic Hodgkin's lymphoma ${ }^{34}$ as well as with a decreased survival in patients with breast cancer $^{35}$ and advanced thyroid cancer. ${ }^{20}$ However, in other malignancies, such as non-small-cell lung cancer, esophageal squamous cell carcinoma, and colon cancer, CD68 ${ }^{+}$macrophage density confers a marked survival advantage. ${ }^{36-38}$ In our study, higher expression of $\mathrm{CD}^{+} 8^{+}$macrophages in NPC specimens predicted better OS and PFS rates and decreased locoregional failure in the univariate Cox regression analysis. As referred above, the CD68 antibody recognizes both M1 and M2 macrophages. ${ }^{17}$ M1 macrophages, which are characterized by the high expressions of proinflammatory cytokines such as interleukin (IL)-1, IL-6, and IL-12, are potent effector cells that kill microorganisms and tumor cells. ${ }^{39}$ In contrast, M2 macrophages, which are characterized by the high expression of IL-4 and IL-10, are prominently involved in cancer initiation, progression, metastasis, angiogenesis, chemoresistance, matrix breakdown, and tumor cell motility. ${ }^{40,41}$ The prognosis related to the higher expression of CD68 may be due to larger proportion or a more active response of M1 cells in the NPC tissues. Despite the nonsignificant $p$-value of the CD68 density after adjustment in the multivariate Cox proportional hazards model, the protective trend for survival still exists. In addition, a study of 60 patients with NPC showed that the CD68 ${ }^{+}$TAM density correlated with a better prognosis. ${ }^{42} \mathrm{CD} 163$ is a highly specific marker of M2 macrophages and has been studied in several aggressive tumors, and the increased expression of 

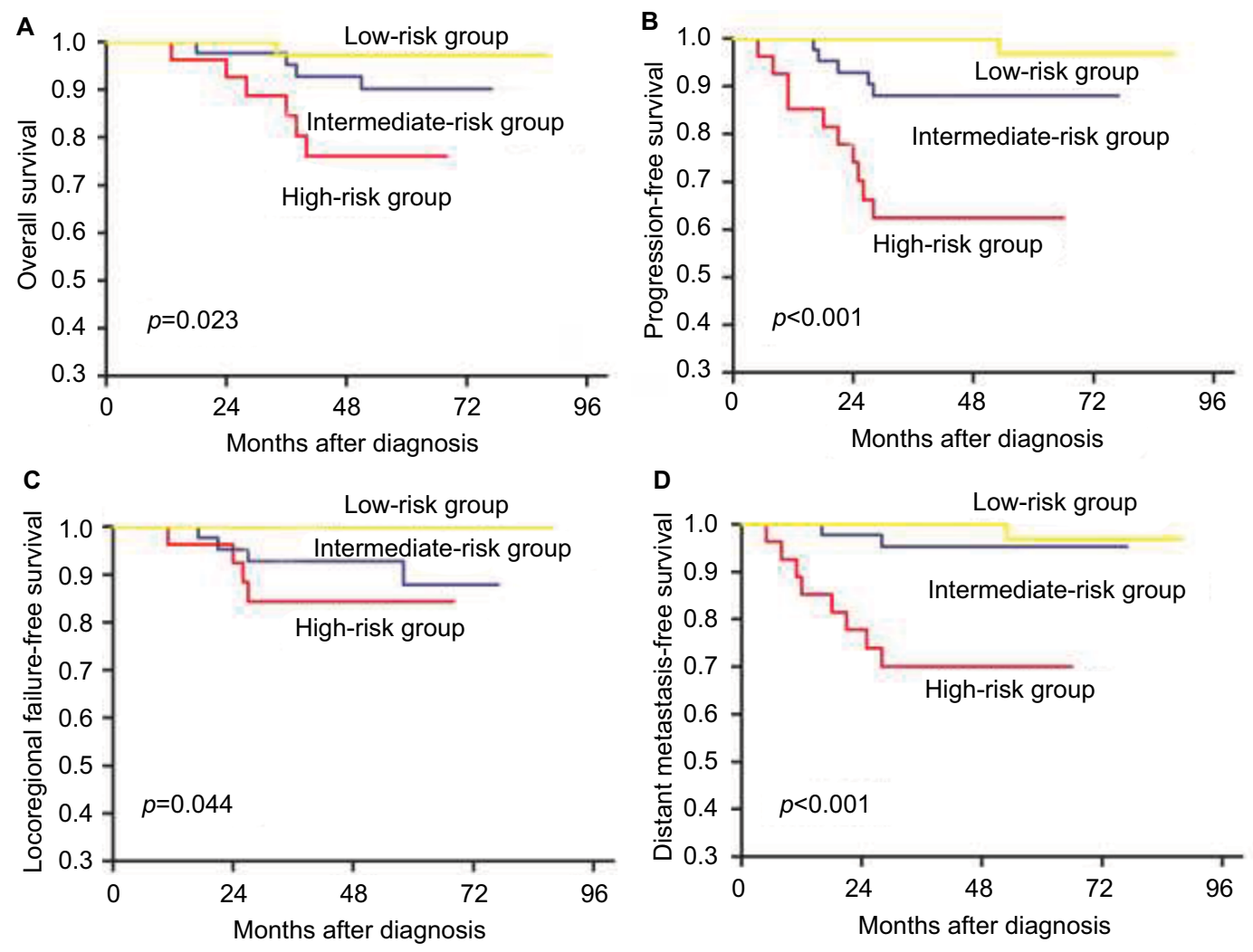

Figure 6 Kaplan-Meier analysis of the 5-year overall survival (A), 5-year progression-free survival (B), 5-year locoregional failure-free survival (C), and the 5-year distant metastasis-free survival (D) in relation to the risk groups of low-risk, intermediate-risk, and high-risk patients with nasopharyngeal carcinoma in the validation test.

CD163 was significantly associated with a poor prognosis in various cancers. ${ }^{23-25,29}$ In our study, higher expression of CD163 predicted worse survival in NPC patients. This result is consistent with previous studies. However, there is controversy about the role of TAMs in tumor progression and metastasis. The individual macrophage markers (ie, CD68, CD163, or CD206) did not correlate with survival in a study on NPC. ${ }^{43}$ The discrepancy may be due to the sample size and keratinizing/non-keratinizing proportions of NPC patients as a regional difference, where 77 (86\%) of the 91 NPC patients had keratinization carcinoma in the Netherlands. More than $95 \%$ of the patients in endemic areas such as southern China were diagnosed with non-keratinizing carcinoma, including those in our study population comprising 260 patients. $^{44}$

Recently, CAF cross talk with cancer cells has been suggested to stimulate tumor progression by creating a favorable microenvironment for progression, invasion, and metastasis. ${ }^{12,14}$ In addition, $\alpha$-SMA immunostaining of CAFs in oral tongue squamous cancer, ${ }^{45}$ esophageal squamous cell carcinoma, ${ }^{46}$ colorectal cancer, ${ }^{47}$ and breast cancer ${ }^{48}$ was related to a poorer prognosis. In our study on NPC, a high level of $\alpha$-SMA expression in CAFs also predicted an adverse prognosis and influenced the OS and PFS mainly through affecting the DMFS independently from the clinical stage. However, in another study on the prognosis of CAFs in 85 NPC patients, besides a poorer prognosis, $\alpha-S M A$ expression in fibroblasts was closely correlated with $\mathrm{T}$ stage $(p<0.05) .{ }^{49}$ This discrepancy may be caused by three reasons. On the one hand, the staging system of the previous study was based on the 2000 World Health Organization criteria; however, all cases in our study were restaged according to the 7 th edition of the UICC/AJCC cancer staging system. The differences between the two staging systems may account for this discrepancy. On the other hand, the population in the training test with 152 patients is much larger than that in the previous study, which may possess a better statistical value.

Many studies in other cancers have described the obvious correlations between the density of CAFs and M2 TAMs. A study on prostate carcinoma reported that the relationship between M2 TAMs and CAFs is reciprocal, as the M2 TAMs affect the resident tissue fibroblast-to-CAF transdifferentiation, leading to their enhanced reactivity. CAFs facilitate monocyte recruitment, macrophage differentiation, and M2 polarization, and then CAFs and M2 TAMs cooperate in promoting tumorigenesis, tumor progression, and metastasis. ${ }^{50}$ The collaboration of CAFs and M2 TAMs in facilitating tumor cell growth and invasion was also verified in bladder cancer, colorectal cancer, breast carcinoma, and 
neuroblastoma. ${ }^{30,32,51,52}$ In addition, in the present study on NPC, besides verifying the correlation between the densities of M2 TAMs and CAFs, we determined that the high expression of both CD163 and $\alpha$-SMA in the primary tumor site results in significantly worse survival in two sets of TMA containing 260 NPC patients, which is highly consistent with previous studies.

The combination of TAM and CAF expression has been developed to predict the outcomes of various cancer patients. In a study on OSCC from Japan, intermediate $\mathrm{CAF}$ expression and high $\mathrm{CD} 163^{+}$macrophage levels were significantly correlated with a poor outcome in patients with OSCC. ${ }^{31}$ According to another study from Spain, the combination of CAF and M2 markers identified three groups of patients with clear differences in disease progression and was regarded as a decisive factor in the survival of advanced-stage patients. ${ }^{30}$ Similarly, based on the density of $\mathrm{CD} 163^{+}$TAMs and CAFs in the tumor stroma, both the cell types are closely related to prognosis, and failure risk groups have been categorized to predict the survival of NPC patients; this stratification is an independent prognostic factor, which may be a good supplement to the TNM classification of NPC patients to identify those with a worse prognosis and consider a more intensive treatment, such as adjuvant chemotherapy and targeted therapy, for those in the high-risk group.

\section{Limitations}

There are some limitations in this study. This is a retrospective design. The patient population's clinical characteristics were diverse; therefore, an observational prospective study is necessary to validate this prognostic model. In addition, the molecular mechanism of the cross talk between CAFs/TAMs and tumor cells in NPC also needs further investigation.

\section{Conclusion}

We identified the densities of CD163+ TAMs and CAFs as independent predictors of survival, and risk groups based on the densities of CD163+ TAM and CAF were independent predictors of survival, which may facilitate patient counseling and individualized treatment. A prospective study to validate this prognostic model is needed.

\section{Availability of data and materials}

The authenticity of this article has been validated by uploading the key raw data onto the Research Data Deposit public platform (http://www.researchdata.org.cn) with the approval number RDDA2018000475.

\section{Acknowledgments}

This work was supported by the National Natural Science Foundation of China (Grant Nos. 81472525, 81572665, 81672680); the International Cooperation Project of Science and Technology Plan of Guangdong Province (Grant Nos. 2014A050503033, 2016A050502011); the Science and Technology Plan Project of Guangdong Province (Grant No. 2013B021800141); and the Foundation of Science and Technology Bureau of Guangzhou City (Grant No. 2014Y2-00179).

\section{Author contributions}

All authors contributed toward data analysis, drafting and revising the paper and agree to be accountable for all aspects of the work.

\section{Disclosure}

The authors report no conflicts of interest in this work.

\section{References}

1. Cao SM, Simons MJ, Qian CN. The prevalence and prevention of nasopharyngeal carcinoma in China. Chin J Cancer. 2011;30(2): 114-119.

2. Wee JT, Ha TC, Loong SL, Qian CN. Is nasopharyngeal cancer really a "Cantonese cancer"? Chin J Cancer. 2010;29(5):517-526.

3. Sarmiento MP, Mejia MB. Preliminary assessment of nasopharyngeal carcinoma incidence in the Philippines: a second look at published data from four centers. Chin J Cancer. 2014;33(3):159-164.

4. Adham M, Kurniawan AN, Muhtadi AI, et al. Nasopharyngeal carcinoma in Indonesia: epidemiology, incidence, signs, and symptoms at presentation. Chin J Cancer. 2012;31(4):185-196.

5. Lee AW, Lau WH, Tung SY, et al. Preliminary results of a randomized study on therapeutic gain by concurrent chemotherapy for regionally-advanced nasopharyngeal carcinoma: NPC-9901 Trial by the Hong Kong Nasopharyngeal Cancer Study Group. J Clin Oncol. 2005;23(28):6966-6975.

6. Lin JC, Jan JS, Hsu CY, Liang WM, Jiang RS, Wang WY. Phase III study of concurrent chemoradiotherapy versus radiotherapy alone for advanced nasopharyngeal carcinoma: positive effect on overall and progression-free survival. J Clin Oncol. 2003;21(4):631-637.

7. Lee AW, Foo W, Mang O, et al. Changing epidemiology of nasopharyngeal carcinoma in Hong Kong over a 20-year period (1980-99): an encouraging reduction in both incidence and mortality. Int $J$ Cancer. 2003;103(5):680-685.

8. Mao YP, Xie FY, Liu LZ, et al. Re-evaluation of 6th edition of AJCC staging system for nasopharyngeal carcinoma and proposed improvement based on magnetic resonance imaging. Int J Radiat Oncol Biol Phys. 2009;73(5):1326-1334.

9. Bissell MJ, Radisky D. Putting tumours in context. Nat Rev Cancer. 2001;1(1):46-54.

10. Feig C, Gopinathan A, Neesse A, Chan DS, Cook N, Tuveson DA. The pancreas cancer microenvironment. Clin Cancer Res. 2012;18(16): $4266-4276$. 
11. Mueller MM, Fusenig NE. Friends or foes - bipolar effects of the tumour stroma in cancer. Nat Rev Cancer. 2004;4(11):839-849.

12. Xing F, Saidou J, Watabe K. Cancer associated fibroblasts (CAFs) in tumor microenvironment. Front Biosci. 2010;15:166-179.

13. Torres S, Garcia-Palmero I, Herrera M, et al. LOXL2 is highly expressed in cancer-associated fibroblasts and associates to poor colon cancer survival. Clin Cancer Res. 2015;21(21):4892-4902.

14. Kalluri R, Zeisberg M. Fibroblasts in cancer. Nat Rev Cancer. 2006;6(5):392-401.

15. Jewett A, Head C, Cacalano NA. Emerging mechanisms of immunosuppression in oral cancers. J Dent Res. 2006;85(12):1061-1073.

16. Mantovani A, Sica A, Locati M. Macrophage polarization comes of age. Immunity. 2005;23(4):344-346.

17. Gottfried E, Kunz-Schughart LA, Weber A, et al. Expression of CD68 in non-myeloid cell types. Scand J Immunol. 2008;67(5):453-463.

18. Chen JJ, Lin YC, Yao PL, et al. Tumor-associated macrophages: the double-edged sword in cancer progression. J Clin Oncol. 2005;23(5): 953-964.

19. Koide N, Nishio A, Sato T, Sugiyama A, Miyagawa S. Significance of macrophage chemoattractant protein-1 expression and macrophage infiltration in squamous cell carcinoma of the esophagus. Am J Gastroenterol. 2004;99(9):1667-1674.

20. Ryder M, Ghossein RA, Ricarte-Filho JC, Knauf JA, Fagin JA. Increased density of tumor-associated macrophages is associated with decreased survival in advanced thyroid cancer. Endocr Relat Cancer. 2008;15(4):1069-1074.

21. Zhu XD, Zhang JB, Zhuang PY, et al. High expression of macrophage colony-stimulating factor in peritumoral liver tissue is associated with poor survival after curative resection of hepatocellular carcinoma. JClin Oncol. 2008;26(16):2707-2716.

22. Costa NL, Valadares MC, Souza PP, et al. Tumor-associated macrophages and the profile of inflammatory cytokines in oral squamous cell carcinoma. Oral Oncol. 2013;49(3):216-223.

23. He KF, Zhang L, Huang CF, et al. CD163+ tumor-associated macrophages correlated with poor prognosis and cancer stem cells in oral squamous cell carcinoma. Biomed Res Int. 2014;2014:838632.

24. Tiainen S, Tumelius R, Rilla K, et al. High numbers of macrophages, especially M2-like (CD163-positive), correlate with hyaluronan accumulation and poor outcome in breast cancer. Histopathology. 2015;66(6):873-883.

25. Kridel R, Xerri L, Gelas-Dore B, et al. The prognostic impact of CD163positive macrophages in follicular lymphoma: a study from the bc cancer agency and the lymphoma study association. Clin Cancer Res. 2015;21(15):3428-3435.

26. Wang S, Sun M, Gu C, et al. Expression of CD163, interleukin-10, and interferon-gamma in oral squamous cell carcinoma: mutual relationships and prognostic implications. Eur J Oral Sci. 2014;122(3):202-209.

27. Shabo I, Stal O, Olsson H, Dore S, Svanvik J. Breast cancer expression of CD163, a macrophage scavenger receptor, is related to early distant recurrence and reduced patient survival. Int J Cancer. 2008;123(4):780-786.

28. Shabo I, Olsson H, Sun XF, Svanvik J. Expression of the macrophage antigen CD163 in rectal cancer cells is associated with early local recurrence and reduced survival time. Int J Cancer. 2009;125(8):1826-1831.

29. Clear AJ, Lee AM, Calaminici M, et al. Increased angiogenic sprouting in poor prognosis $\mathrm{FL}$ is associated with elevated numbers of CD163+ macrophages within the immediate sprouting microenvironment. Blood. 2010;115(24):5053-5056.

30. Herrera M, Herrera A, Dominguez G, et al. Cancer-associated fibroblast and M2 macrophage markers together predict outcome in colorectal cancer patients. Cancer Sci. 2013;104(4):437-444.

31. Fujii N, Shomori K, Shiomi T, et al. Cancer-associated fibroblasts and CD163-positive macrophages in oral squamous cell carcinoma: their clinicopathological and prognostic significance. J Oral Pathol Med. 2012;41(6):444-451.

32. Hashimoto O, Yoshida M, Koma Y, et al. Collaboration of cancerassociated fibroblasts and tumour-associated macrophages for neuroblastoma development. J Pathol. 2016;240(2):211-223.
33. Xie D, Sham JS, Zeng WF, et al. Heterogeneous expression and association of beta-catenin, p16 and c-myc in multistage colorectal tumorigenesis and progression detected by tissue microarray. Int $J$ Cancer. 2003;107(6):896-902.

34. Steidl C, Lee T, Shah SP, et al. Tumor-associated macrophages and survival in classic Hodgkin's lymphoma. $N$ Engl J Med. 2010;362(10):875-885.

35. Medrek C, Ponten F, Jirstrom K, Leandersson K. The presence of tumor associated macrophages in tumor stroma as a prognostic marker for breast cancer patients. BMC Cancer. 2012;12:306.

36. Welsh TJ, Green RH, Richardson D, Waller DA, O’Byrne KJ, Bradding P. Macrophage and mast-cell invasion of tumor cell islets confers a marked survival advantage in non-small-cell lung cancer. J Clin Oncol. 2005;23(35):8959-8967.

37. Li J, Zhang BZ, Qin YR, et al. CD68 and interleukin 13, prospective immune markers for esophageal squamous cell carcinoma prognosis prediction. Oncotarget. 2016;7(13):15525-15538.

38. Forssell J, Oberg A, Henriksson ML, Stenling R, Jung A, Palmqvist R. High macrophage infiltration along the tumor front correlates with improved survival in colon cancer. Clin Cancer Res. 2007;13(5):1472-1479.

39. Benoit M, Desnues B, Mege JL. Macrophage polarization in bacterial infections. J Immunol. 2008;181(6):3733-3739.

40. Mantovani A, Sozzani S, Locati M, Allavena P, Sica A. Macrophage polarization: tumor-associated macrophages as a paradigm for polarized M2 mononuclear phagocytes. Trends Immunol. 2002;23(11): 549-555.

41. Sugimura K, Miyata H, Tanaka K, et al. High infiltration of tumorassociated macrophages is associated with a poor response to chemotherapy and poor prognosis of patients undergoing neoadjuvant chemotherapy for esophageal cancer. J Surg Oncol. 2015;111(6): 752-759.

42. Peng J, Ding T, Zheng LM, Shao JY. [Influence of tumor-associated macrophages on progression and prognosis of nasopharyngeal carcinoma]. Ai Zheng. 2006;25(11):1340-1345.

43. Ooft ML, van Ipenburg JA, Sanders ME, et al. Prognostic role of tumour-associated macrophages and regulatory $\mathrm{T}$ cells in EBVpositive and EBV-negative nasopharyngeal carcinoma. J Clin Pathol. 2018;71(3):267-274.

44. Wang HY, Chang YL, To KF, et al. A new prognostic histopathologic classification of nasopharyngeal carcinoma. Chin J Cancer. 2016;35:41.

45. Li H, Zhang J, Chen SW, et al. Cancer-associated fibroblasts provide a suitable microenvironment for tumor development and progression in oral tongue squamous cancer. J Transl Med. 2015;13:198.

46. Ha SY, Yeo SY, Xuan YH, Kim SH. The prognostic significance of cancer-associated fibroblasts in esophageal squamous cell carcinoma. PLoS One. 2014;9(6):e99955.

47. Tsujino T, Seshimo I, Yamamoto H, et al. Stromal myofibroblasts predict disease recurrence for colorectal cancer. Clin Cancer Res. 2007;13(7):2082-2090.

48. Yamashita M, Ogawa T, Zhang X, et al. Role of stromal myofibroblasts in invasive breast cancer: stromal expression of alpha-smooth muscle actin correlates with worse clinical outcome. Breast Cancer. 2012;19(2):170-176.

49. Chen J, Yang P, Xiao Y, et al. Overexpression of alpha-sma-positive fibroblasts (CAFs) in nasopharyngeal carcinoma predicts poor prognosis. $J$ Cancer. 2017;8(18):3897-3902.

50. Comito G, Giannoni E, Segura CP, et al. Cancer-associated fibroblasts and M2-polarized macrophages synergize during prostate carcinoma progression. Oncogene. 2014;33(19):2423-2431.

51. Rama-Esendagli D, Esendagli G, Yilmaz G, Guc D. Spheroid formation and invasion capacity are differentially influenced by co-cultures of fibroblast and macrophage cells in breast cancer. Mol Biol Rep. 2014;41(5):2885-2892.

52. Miyake M, Hori S, Morizawa Y, et al. CXCL1-mediated interaction of cancer cells with tumor-associated macrophages and cancer-associated fibroblasts promotes tumor progression in human bladder cancer. Neoplasia. 2016;18(10):636-646. 


\section{Publish your work in this journal}

Cancer Management and Research is an international, peer-reviewed open access journal focusing on cancer research and the optimal use of preventative and integrated treatment interventions to achieve improved outcomes, enhanced survival and quality of life for the cancer patient. The manuscript management system is completely online and includes a very quick and fair peer-review system, which is all easy to use. Visit http://www.dovepress.com/testimonials.php to read real quotes from published authors.

Submit your manuscript here: https://www.dovepress.com/cancer-management-and-research-journal 